UCRL-JC-130865

PREPRINT

\title{
Overview of the WARP Code and Studies of Transverse Resonance Effects
}

\author{
A. Friedman \\ This paper was prepared for submittal to the \\ Workshop on Space Charge Physics in High Intensity \\ Long Island, NY \\ May 4-7, 1998
}

May 15, 1998

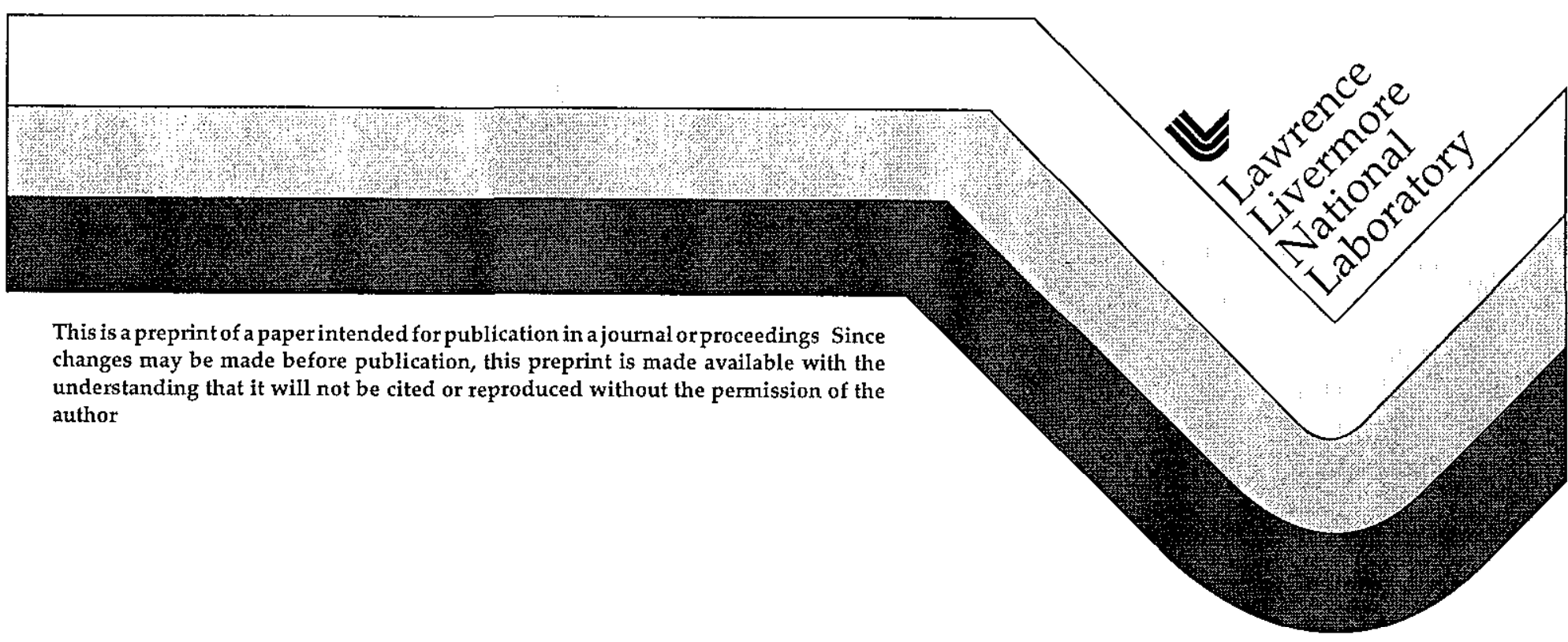




\section{DISCLAIMER}

This document was prepared as an account of work sponsored by an agency of the United States Government. Neither the United States Government nor the University of California nor any of their employees, makes any warranty, express or implied, or assumes any legal liability or responsibility for the accuracy, completeness, or usefulness of any information, apparatus, product, or process

disclosed, or represents that its use would not infringe privately owned rights. Reference herein to any specific commercial product, process, or service by trade name, trademark, manufacturer, or otherwise, does not necessarily constitute or imply its endorsement, recommendation, or favoring by the United States Government or the University of California. The views and opinions of authors expressed herein do not necessarily state or reflect those of the United States Government or the University of California, and shall not be used for advertising or product endorsement purposes. 


\title{
Overview of the WARP Code and Studies of Transverse Resonance Effects*
}

\author{
Alex Friedman \\ Lawrence Livermore National Laboratory, Livermore, CA 94551, USA
}

\begin{abstract}
Two papers presented at the Shelter Island workshop are very briefly summarized here, in view of recent publications elsewhere The WARP code, developed for Heavy-Ion beam-driven inertial confinement Fusion (HIF) accelerator studies, combines features of a particle-in-cell plasma simulation and an accelerator tracking program. Its methods and architecture have been developed for efficiency both in detailed simulation of individual machine sections and in long-time beam tracking. The transverse "slice" model in the code has been applied to the study of transverse resonance effects associated with quadrupole strength errors. These simulations confirm that rapid passage through a resonance can reduce the associated mismatch and emittance growth References to published details and to other sources of information are supplied.
\end{abstract}

\section{WARP CODE OVERVIEW}

WARP is a particle-in-cell $(1,2)$ beam simulation code offering 3-D, axisymmetric $(r, z)$, and transverse $(x, y)$ geometries. The code was developed for Heavy-Ion beamdriven inertial confinement Fusion (HIF) accelerator studies (3-8). In this application, and particularly in the U.S approach based on induction technology, space charge forces dominate over the beam thermal pressure

WARP (9-14) incorporates detailed descriptions of various acceletator and beamline elements, and was designed to follow beams efficiently over long path lengths A hierarchy of models affords increasing levels of detail in the description of the accelerator lattice While some of these models are based on an expansion in powers of the off-axis separation, the code can use models which are good to "all orders" (such as field specification on a full 3-D grid) when sufficient knowledge of the field is available Several Poisson equation solvers are available, optionally incorporating internal conducting elements from "first principles" with subgrid-scale resolution of the conductor boundary locations

WARP uses time (rather than path length) as the independent coordinate for particle motion, this facilitates the treatment of longitudinally-extended beams The code gets its name from its use of "warped" coordinates, that is, a sequence of Cartesian grid sections aligned with the local beamline (so that each lattice element is described in its own natural coordinate system) linked by sections of "polar coordinate" grid. Coordinate transformations account for the bends while preserving the symplectic nature of the underlying advance The fields from neighboring elements can overlap, even when 
those elements are separated by a bend and so are described with reference to different frames The user specifies a coordinate system which describes the beamline as it is laid out "in the laboratory," and need not specify a reference orbit In the 3-D and r,z models, no paraxial approximation is invoked.

The code offers an interactive, interpreter-driven user interface for code steering and scripting-language control This capability allows significant changes in the code's operation to be effected by the user without requiring recompilation. It provides a rich simulation environment which offers ready access to the code's internal database, algebraic transformations, and interactive visualization WARP has been parallelized to run on supercomputers, symmetric multiprocessors, and workstation clusters using message-passing techniques While already quite capable, the code is still under development, it has not yet "settled down" to a version suitable for unlimited release

\section{TRANSVERSE RESONANCE EFFECTS}

In the recirculating induction accelerator approach to an $\mathrm{HIF}$ driver $(15,16)$, the beam is rapidly accelerated "through resonances" - it is "different on every lap" and errors add much as in a long linac Beam steering is used to correct the centroid motion In some RF linac / storage ring approaches, resonance crossing is driven by rapid bunching of the beam, which shifts the resonances as a result of the increasing space charge density and image effects We are carrying out studies (17) of transverse resonance effects in rings with space charge The beams we consider are highly spacecharge-dominated, with an undepressed phase advance per lattice period of $\sigma_{0} \sim 75^{\circ}$ reduced by the transverse self-fields to $\sigma \sim 15-20^{\circ}$. These parameters are similar to those of the "small recirculator" (16) under development at LLNL We consider periodic quadrupole strength errors which can resonantly drive symmetric and antisymmetric mismatch oscillations of the beam core. Interactions with those core oscillations can drive particles into the halo and degrade the emittance Using a 2D transverse "slice" model in the WARP PIC code, we study how aggressive acceleration or bunching, leading to rapid passage through a single applied resonance, affects the emittance Power spectra are computed and their many features identified We confirm that rapid passage through the resonance significantly reduces the attendant emittance growth The growth rate is seen to be strongly correlated with the instantaneous degree of mismatch.

As part of this study, the convergence of the PIC method employed was investigated Runs using 20,000 particles showed some jaggedness of particle orbits and enhanced emittance growth (resulting from the nonphysically-large collisionality associated with the small particle number), but were gener ally deemed reliable enough for studies such as these Runs using 80,000 and 320,000 particles were well-behaved, and produced essentially converged results

\section{ACKNOWLEDGMENTS}

*This work was performed under the auspices of the U.S Department of Energy by LLNL under contract W-7405-ENG-48.

The author gratefully acknowledges the contributions of his colleagues and most frequent co-authors, John J Barnard, Debra A Callahan-Miller, David P Grote, Irving Haber, and Steven M Lund, to the work listed in the References. 


\section{REFERENCES}

1 C K Birdsall and A B Langdon, Plasma Physics via Computer Simulation, ISBN 007-005371-5, Adam Hilger / IOP, Bristol, 1991

2 R. W Hockney and J W. Eastwood, Computer Simulation Using Particles, ISBN 085274-392-0, Adam Hilger / IOP, Bristol, 1988

3. The U S Heavy Ion Fusion World-Wide Web pages can be found at http //fusion lbl gov/; see also the links and references therein

4 Energy from Inertial Fusion, STI/PUB/944, ISBN 92-0-100794-9, W J Hogan, scientific editor, IAEA, Vienna, 1995

5 Proceedings of the 10th International Symposium on Heavy Ion Inertial Fusion (Frascati) Nuovo Cimento 106 A Nos 11-12 (1994)

6 Proceedings of the 11th International Symposium on Heavy Ion Inertial Fusion (Princeton). Fusion Engineering and Design 32-33 (1996)

7 Proceedings of the 12th International Symposium on Heavy Ion Inertial Fusion (Heidelberg) Nuclear Instruments and Methods A, in press (1998)

8 A Friedman, "Induction-Accelerator Heavy-Ion Fusion Status and Beam Physics Issues," Proc IUPAP / Int 'l Committee for Future Accelerators' Workshop on Space Charge Dominated Beams and Applications of High Brightness Beams, Bloomington IN, Oct 10-13, 1995 (S Y. Lee, Ed, AIP Conference Proceedings No 377, 1996)

9 A Friedman, D P Grote, and I Haber, "Three-dimensional particle simulation of heavy-ion fusion beams," Phys Fluids B Plasma Physics 4, 2203 (1992)

10 D P Grote, "Methods Used in WARP3d, a Three Dimensional PIC/Accelerator Code," Proceedings of the Computational Accelerator Physics Conference, 1996, AIP conference proceedings 391, pp 51-58

11 D P Grote, A Friedman, I Haber, and S S Yu, "Three-Dimensional Simulations of High Current Beams in Induction Accelerators with WARP3d", Fusion Engineering and Design 32, 193 (1996)

12 S M Lund et al, "Numerical Simulation of Intense-Beam Experiments at LLNL and LBNL," Proc 12th International Symposium on Heavy-Ion Inertial Fusion, Heidelberg Germany, September 23-27,1997, accepted for publication in Nuclear Instruments and Methods A, 1998

13 D P Grote I Haber, A Friedman, and S M Lund, "WARP3d, A Three-Dimensional PIC Code For High-Current Ion-Beam Propagation Developed For Heavy-Ion Fusion," Lawrence Livermore National Laboratory Quarterly Report for Fourth Quarter 1996, available on the Web at http //lasers llnl gov/lasers/pubs/jul-sep96/ju196 html

14 D P Grote, A Friedman, I Haber, W Fawley, and J -L Vay, "New Developments in WARP Progress Toward End-to-End Simulation," Proc 12th International Symposium on Heavy-Ion Inertial Fusion, Heidelberg Germany, September 23-27,1997, accepted for publication in Nuclear Instruments and Methods A, 1998

15 J J Barnard, F J Deadrick, A Friedman, D P Grote, L V Griffith, H C Kirbie, V K Neil, M A Newton, A C Paul, W M Sharp, H D Shay, R O Bangerter, A Faltens, C G Fong, D L Judd, E. P Lee, L L Reginato, S S Yu, and T F Godlove, "Recirculating Induction Accelerators as Drivers for Heavy Ion Fusion," Physics of Fluids B Plasma Physics 5, 2698 (1993)

16 A Friedman et al, "Progress Toward a Prototype Recirculating Ion Induction Accelerator," Lawrence Livermore National Laboratory Quarterly Report for Api il-June 1995, available on the Web at

http //lasers llnl gov/lasers/pubs/apr-june95/Friedman pdf

17 A Friedman, J J Barnard, D P Grote, and I Haber, Simulation Studies of Transverse Resonance Effects in Space-Charge Dominated Beams, Proc 12th International Symposium on Heavy-Ion Inertial Fusion, Heidelberg Germany, September 23-27,1997, accepted for publication in Nuclear Instruments and Methods A, 1998 


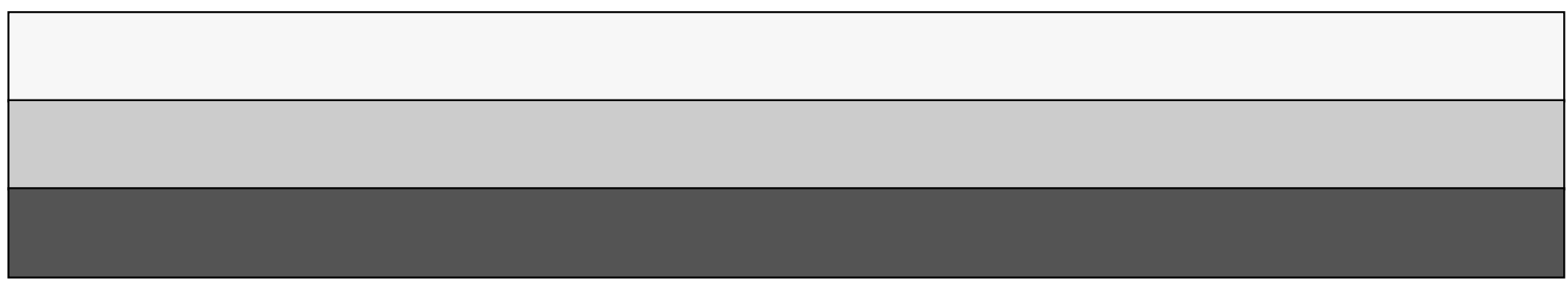

